\title{
Perfil clínico-epidemiológico dos acidentes ofídicos ocorridos na ilha de Colares, Pará, Amazônia oriental
}

\section{Clinical-epidemiological profile of snakebites occurred on the island of Colares, Pará, eastern Amazonia}

\author{
Cláudio Douglas de Oliveira Guimarães ${ }^{1}$; Maria Correia Palha²; Jean carlos Ramos Silva ${ }^{3}$
}

\section{Resumo}

Descrever o perfil clínico-epidemiológico dos acidentes ofídicos ocorridos na ilha de Colares, Pará, Brasil. O estudo consistiu em uma investigação epidemiológica transversal a partir da análise de todos os casos de envenenamento por serpentes peçonhentas atendidos no Hospital Municipal e notificados ao Sistema Nacional de Notificações e Agravos (SINAN) do Ministério da Saúde, entre janeiro de 2007 e dezembro de 2011. Os dados obtidos a partir das fichas de notificação foram analisados utilizando-se estatística descritiva simples no Microsoft Excel (versão 2007). Foram notificados ao SINAN 94 casos de acidentes ofídicos, sendo observada maior concentração dos envenenamentos no primeiro semestre de cada ano, período em que ocorrem os maiores índices pluviométricos para a região. A maioria dos acidentes aconteceu na zona rural $(85,11 \%)$, durante a realização dos trabalhos braçais $(55,32 \%)$, com maior incidência em homens ( $73,40 \%$ ), predominantemente provocados por serpentes do gênero Bothrops $(69,62 \%)$. Foram identificadas falhas no protocolo de soroterapia, sendo verificada sua utilização em dois casos cujas serpentes não eram peçonhentas e não administrado em dois casos confirmados de acidentes ofídicos envolvendo serpentes peçonhentas. O pé foi a região mais acometida $(51,06 \%)$ e a dor, edema e manifestações vagais foram os sinais mais frequentes. Estes resultados demonstram que o município de Colares segue o perfil dos acidentes ofídicos de outras localidades rurais amazônicas, sendo necessária maior atenção dos profissionais de saúde quanto ao preenchimento das fichas de notificação e aos protocolos terapêuticos dispensados às vítimas.

Palavras-chave: Envenenamento. Ofidismo. Saúde pública. Serpentes. Bothrops

\begin{abstract}
To describe the clinical and epidemiological profile of snakebites occurred on the island of Colares, Pará, Brazil. The study consisted of a cross-epidemiological research based on the analysis of all cases of poisoning by venomous snakes treated at City Hospital and served on the National System Notifications and Disability (SINAN) and conducted to the Ministry of Health between January 2007 and December 2011. Data obtained from the notification records were analyzed using simple descriptive statistics in Microsoft Excel (2007 version). Ninety-four cases of snakebites were reported to SINAN and the highest frequency occurred in the first half of each year, a period in which most precipitation occurs in the region. Most accidents occurred in rural areas (85.11\%), while performing manual labor
\end{abstract}

\footnotetext{
${ }^{1}$ Mestre em Saúde e Produção Animal na Amazônia pela Universidade Federal Rural da Amazônia - UFRA - Pesquisador Associado do Projeto Bio-Fauna - Instituto Socioambiental e dos Recursos hídricos - UFRA - Email: cdoguimaraes@gmail.com

${ }^{2}$ Doutora em Ciências Biológicas pela Universidade Federal de Pernambuco. Professora Adjunta da Universidade Rural da Amazônia Coordenadora do Projeto Bio-Fauna - Instituto Socioambiental e dos Recursos hídricos - UFRA - Email: faunaufra@gmail.com

${ }^{3}$ Pós-Doutorado em Saúde Animal pela Universidade de São Paulo - USP. Professor Associado I do Departamento de Medicina Veterinária da Universidade Federal Rural de Pernambuco - UFRPE - Email: jcrsilva16@gmail.com
} 
$(55.32 \%)$, with higher incidence in men $(73.40 \%)$, with predominance of accidents caused by snakes of the genus (Bothrops) (69.62\%). Failures were identified in the protocol of serum therapy, as it was used in two cases in which the snakes were not venomous and not used in two other cases involving poisonous snakes. Regarding clinical aspects, the foot was the most affected region $(51.06 \%)$ while pain, edema and vagal manifestations were the most frequent signs. These results demonstrate that the municipality of necklaces follows the profile of snakebites other Amazonian rural areas. Greater attention is needed from health professionals when filling in the notification forms and when following therapeutic protocols of snakebite victims.

Keywods: Poisoning. Snakebite. Public health. Snakes. Bothrops.

\section{Introdução}

Os acidentes ofídicos são um importante problema de saúde pública, especialmente para aqueles povos que habitam países tropicais (MISE; LIRA-DA-SILVA; CARVALHO, 2007; MORENO et al., 2005), regiões onde há maior concentração destes acidentes (GUTIÉRREZ; THEAKSTON; WARREL, 2006). No Brasil, os envenenamentos por serpentes representam aproximadamente 29.000 casos por ano e uma média de 125 óbitos (BERNARDE, 2014). Estas estimativas, entretanto, encontram-se subestimadas pelas dificuldades de registro dos acidentes nas regiões mais remotas do país, em especial na região Centro-Oeste, Nordeste e Norte (BRASIL, 2001). Esta última merece destaque devido à maior incidência de casos por habitante, principalmente em localidades da região amazônica (ARAÚJO; SANTALUCIA; CABRAL, 2003; WALDEZ; VOGT, 2009). Nesta região, a maioria dos casos de envenenamento registrados deve-se à serpente Bothrops atrox (CAMPBELL; LAMAR, 2004) e os trabalhadores das áreas rurais são as principais vítimas (BOCHNER; STRUCHINER, 2004; MORENO et al., 2005).

Alguns fatores socioambientais como tempo chuvoso e quente, vegetação, habitação rural e processo de urbanização de áreas periféricas das cidades, estão diretamente relacionados aos índices de acidentes ofídicos (MORENO et al., 2005; PINHO; OLIVEIRA; FALEIROS, 2004). Além disso, uma economia predominantemente agropastoril/extrativista e atividades de lazer, como caça e pesca - muito presentes em comunidades rurais amazônicas - frequentemente em áreas de mata nativa, concorrem para a maior exposição da população à fauna ofídica e, consequentemente, aos acidentes com serpentes peçonhentas (MORENO et al., 2005). No entanto, apesar da importância dos acidentes ofídicos para a saúde coletiva, aspectos relacionados à pesquisa epidemiológica, acesso ao tratamento e à qualificação de profissionais em saúde ainda são negligenciados pelas políticas públicas nacionais (GUTIÉRREZ; THEAKSTON; WARREL, 2006, GUTIÉRREZ et al., 2007).

Sabe-se que os estudos epidemiológicos dos acidentes ofídicos são importantes para a garantia de melhores condições de atendimento e tratamento aos acidentados, reduzindo, dessa forma, a gravidade e a letalidade que esses acidentes podem provocar (LIMA; CAMPOS; RIBEIRO, 2009). Porém, mesmo sendo um agravo de notificação obrigatória, os dados disponíveis não retratam a real magnitude desse problema, provavelmente devido à subnotificação dos casos, tendo em vista, entre outros fatores, as dificuldades de acesso aos serviços de saúde da região amazônica (BOCHNER; STRUCHINER, 2003). Este estudo foi conduzido com o objetivo de caracterizar o perfil clínicoepidemiológico dos acidentes ofídicos ocorridos na ilha de Colares, Pará, Amazônia oriental.

\section{Material e Métodos}

Esta pesquisa consistiu em um estudo epidemiológico transversal de todos os casos de acidentes ofídicos atendidos no Hospital Municipal de Colares e notificados ao Sistema Nacional de 
Notificação e Agravos (SINAN) do Ministério da Saúde, entre janeiro de 2007 e dezembro de 2011. Os dados foram obtidos a partir de documentação indireta (fichas de notificação) disponibilizada pelo serviço de saúde do município, sendo consideradas informações sociodemográficas (sexo, idade, ocupação, zona de moradia), epidemiológicas (data, zona de ocorrência, tempo decorrido entre a picada e o atendimento, circunstância do acidente), clínicas (local da picada, manifestações locais/sistêmicas, classificação do caso, soroterapia, evolução do caso) e biológicas (serpente envolvida) dos acidentes.

O estudo foi realizado no município de Colares, que é uma ilha pertencente à mesorregião do Nordeste Paraense (Costa oriental) com uma área de aproximadamente $612,5 \mathrm{~km}^{2}$, distando 93,9 $\mathrm{km}$ de Belém, capital do estado do Pará (IDESP, 2013). Apresenta temperaturas elevadas, com média de $26^{\circ} \mathrm{C}$, umidade relativa do ar de $82 \%$ e extensa rede hidrográfica com rios e igarapés. Possui uma população estimada em 11.382 habitantes, com a maioria da população $(70 \%)$ residindo na zona rural dividida em 22 localidades (vilas e povoados) (IDESP, 2013; SILVA et al., 1999a, 1999b). Os dados deste estudo foram planificados e analisados empregandose estatística descritiva simples no Microsoft Excel (versão 2007).

\section{Resultados}

No presente estudo, constatou-se que 94 notificações de acidentes envolvendo serpentes peçonhentas na ilha de Colares foram encaminhadas ao SINAN, sendo observado um ligeiro aumento da incidência de casos ao longo do período considerado (janeiro de 2007 a dezembro de 2011), com maior frequência de acidentes ocorridos no ano de 2009. Ao realizar a distribuição mensal das notificações, verificou-se maior concentração dos casos de acidentes ofídicos no primeiro semestre (58,51\%; $n=55)$ em relação ao segundo $(41,49 \%$; $n=39)$, sendo observada maior frequência nos meses de janeiro $(15,94 \% ; n=15)$ e agosto $(15,94 \% ; n=15)$ (Tabela 1$)$.

Tabela 1 - Distribuição anual e mensal dos 94 casos de acidentes ofídicos ocorridos na Ilha de Colares PA, de janeiro de 2007 a dezembro de 2011 notificados ao SINAN

\begin{tabular}{ccccccccccccccc}
\hline \multirow{2}{*}{ Ano } & \multicolumn{10}{c}{ Mês de ocorrência } \\
\cline { 2 - 14 } & Jan & Fev & Mar & Abr & Mai & Jun & Jul & Ago & Set & Out & Nov & Dez & Total \\
\hline $\mathbf{2 0 0 7}$ & 4 & 0 & 1 & 1 & 1 & 2 & 0 & 2 & 1 & 0 & 3 & 0 & 15 \\
$\mathbf{2 0 0 8}$ & 3 & 2 & 1 & 2 & 1 & 2 & 0 & 3 & 2 & 1 & 1 & 1 & 19 \\
$\mathbf{2 0 0 9}$ & 4 & 4 & 1 & 1 & 3 & 1 & 1 & 5 & 2 & 0 & 0 & 2 & 24 \\
$\mathbf{2 0 1 0}$ & 1 & 2 & 3 & 1 & 0 & 0 & 1 & 3 & 2 & 2 & 0 & 1 & 16 \\
$\mathbf{2 0 1 1}$ & 3 & 2 & 1 & 5 & 2 & 1 & 2 & 2 & 1 & 0 & 1 & 0 & 20 \\
Total & 15 & 10 & 7 & 10 & 7 & 6 & 4 & 15 & 8 & 3 & 5 & 4 & 94 \\
\hline
\end{tabular}

Fonte: Autores.

De acordo com a análise dos dados, a maioria dos acidentes ocorreu durante a realização do trabalho $(55,32 \% ; n=52)$ com os homens sendo mais acometidos $(73,40 \% ; n=69)$ (Tabela 2$)$ e maior número de ocorrências identificadas na zona rural $(85,11 \% ; n=80)$. 
Tabela 2 - Características socioeconômicas dos acidentados envolvidos nos 94 casos de acidentes ofídicos ocorridos na Ilha de Colares - PA, de janeiro de 2007 a dezembro de 2011 e notificados ao SINAN

\begin{tabular}{|c|c|c|}
\hline Parâmetro & Valor absoluto & Percentual \\
\hline \multicolumn{3}{|l|}{ Sexo } \\
\hline Masculino & 69 & $73,40 \%$ \\
\hline Feminino & 25 & $26,60 \%$ \\
\hline Idade Média em anos (desvio padrão) & \multicolumn{2}{|c|}{$33(14,03)$} \\
\hline \multicolumn{3}{|l|}{ Escolaridade } \\
\hline Analfabeto & 2 & $2,13 \%$ \\
\hline Fundamental incompleto & 57 & $60,64 \%$ \\
\hline Fundamental completo & 7 & $7,45 \%$ \\
\hline Médio incompleto & 7 & $7,45 \%$ \\
\hline Médio completo & 7 & $7,45 \%$ \\
\hline Superior completo & 1 & $1,06 \%$ \\
\hline Ignorado/ Não se aplica & 13 & $13,83 \%$ \\
\hline \multicolumn{3}{|l|}{ Ocupação } \\
\hline Não informado & 47 & $50,02 \%$ \\
\hline Estudante & 19 & $20,21 \%$ \\
\hline Agricultor & 12 & $12,77 \%$ \\
\hline Doméstica & 6 & $6,38 \%$ \\
\hline Pescador & 3 & $3,19 \%$ \\
\hline Lavador de carro & 2 & $2,13 \%$ \\
\hline Aposentado & 1 & $1,06 \%$ \\
\hline Mão-de-obra & 1 & $1,06 \%$ \\
\hline Vigia & 1 & $1,06 \%$ \\
\hline Agente comunitário de saúde & 1 & $1,06 \%$ \\
\hline Extrativista & 1 & $1,06 \%$ \\
\hline
\end{tabular}

Fonte: Autores.

Em relação à ocupação das vítimas, verificou-se que a maioria dos acidentes ocorreu entre estudantes $(20,21 \% ; n=19)$. No entanto, vale ressaltar que metade das fichas encaminhadas ao SINAN não apresentava o registro da ocupação dos acidentados (Tabela 2) e, pela representatividade do percentual, deve ser levada em consideração, pois pode influenciar na análise dessa variável.

Nestapesquisa, adultos emidade economicamente ativa (21 a 40 anos) foram mais acometidos (43,62\%; $\mathrm{n}=41$ ), seguidos de crianças e jovens ( 0 a 20 anos) $(25,53 \% ; n=24)$, adultos (41 - 60 anos) $(20,21 \%$; $\mathrm{n}=19)$ e idosos $(61-80$ anos $)(10,64 \% ; n=10)$.

Do total de 94 casos de acidentes ofídicos, apenas 79 acidentes tiveram a identificação da serpente informada com dois casos de acidentes envolvendo serpentes não peçonhentas e 11 casos cuja informação foi ignorada. Das fichas que continhas essa informação completa $(n=79)$, foi observada predominância de envenenamentos causados pelo gênero Bothrops $(69,62 \% ; n=55)$ seguida pelo gênero Lachesis $(30,38 \%$; $\mathrm{n}=24)$.

Os resultados do presente estudo apontam para um atendimento relativamente rápido, pois na maioria dos casos $(61,70 \% ; n=58)$ o tempo decorrido entre a picada até o atendimento ambulatorial foi inferior a uma hora, fato que pode ter contribuído para o sucesso do tratamento. No entanto, foram identificadas falhas quanto à instituição da soroterapia, como por exemplo, a utilização do soro antiofídico em apenas $92(97,87 \%)$ casos, o uso de soro antibotrópico (SAB) em três casos de acidentes laquéticos, o uso de soro antibotropicolaquético (SABL) em dois casos de acidentes por serpentes não peçonhentas e a não utilização de soro específico em um caso de acidente botrópico (Tabela 3). 
Tabela 3 - Distribuição dos 94 casos de acidentes ofídicos ocorridos na Ilha de Colares - PA, de janeiro de 2007 a dezembro de 2011 notificados ao SINAN quanto à soroterapia instituída

\begin{tabular}{|c|c|c|c|c|c|c|c|c|c|c|}
\hline & \multicolumn{2}{|c|}{ SAB } & \multicolumn{2}{|c|}{ SABL } & \multicolumn{2}{|c|}{ SABC } & \multicolumn{2}{|c|}{ Nenhum } & \multicolumn{2}{|c|}{$\mathbf{N}^{0}$ de casos } \\
\hline & V.A. & $\%$ & V.A. & $\%$ & V.A. & $\%$ & V.A. & $\%$ & V.A. & $\%$ \\
\hline Acidente botrópico & 45 & 47,87 & 9 & 9,57 & 0 & 0,00 & 1 & 1,06 & 55 & 58,51 \\
\hline Acidente laquético & 3 & 3,19 & 21 & 22,34 & 0 & 0,00 & 0 & 0,00 & 24 & 25,53 \\
\hline Não peçonhenta & 0 & 0,00 & 2 & 2,13 & 0 & 0,00 & 0 & 0,00 & 2 & 2,13 \\
\hline Informação ignorada & 5 & 5,32 & 5 & 5,32 & 0 & 0,00 & 1 & 1,06 & 11 & 11,70 \\
\hline Informação não preenchida & 1 & 1,06 & 0 & 0,00 & 1 & 1,06 & 0 & 0,00 & 2 & 2,13 \\
\hline Total & 54 & 57,45 & 37 & 39,36 & 1 & 1,06 & 2 & 2,13 & 94 & 100 \\
\hline
\end{tabular}

SAB - soro antibotrópico; SABL - soro antibotropicolaquético; SABC - soro antibotropicocrotálico; V.A. - valor absoluto

Fonte: Autores.

Ainda com relação à soroterapia, considerando o número de ampolas prescritas e o tipo de acidente, verificou-se que em todos os casos de acidente laquético comprovados ou, pelo menos, suspeitos, a média de ampolas foi sete, número abaixo do que é recomendado pelo ministério da saúde (10 ampolas).

Quanto à gravidade do caso, houve maior número de registros de grau leve, com um total de 59 $(62,77 \%)$ casos, seguido pelo grau moderado com $29(30,85 \%)$ casos, e um (1,06\%) caso considerado grave. Apenas um $(1,06 \%)$ caso foi ignorado e quatro $(4,26 \%)$ casos não apresentaram registro dessa informação.

Em relação à região anatômica acometida durante os acidentes ofídicos, os membros inferiores foram os mais atingidos, sendo o pé e a perna as áreas mais afetadas, como mostra a Tabela 5. Na maioria dos casos, as manifestações locais foram as alterações clínicas mais presentes $(92,55 \%$; $n=87)$, das quais dor e edema foram os sinais mais frequentes. Em contrapartida, as alterações sistêmicas foram observadas em 4,26\% ( $\mathrm{n}=4)$ dos casos, sendo as manifestações vagais as mais frequentes (Tabela 6).

Tabela 5 - Região anatômica acometida nos acidentes ofídicos ocorridos na Ilha de Colares - PA, de janeiro de 2007 a dezembro de 2011 notificados ao SINAN

\begin{tabular}{ccc}
\hline Local da picada & Valor absoluto & Percentual \\
\hline Pé & 48 & $51,06 \%$ \\
Perna & 15 & $15,96 \%$ \\
Dedo da mão & 11 & $11,70 \%$ \\
Mão & 7 & $7,45 \%$ \\
Braço & 5 & $5,32 \%$ \\
Dedo do pé & 4 & $4,26 \%$ \\
Antebraço & 3 & $3,19 \%$ \\
Tronco & 1 & $1,06 \%$ \\
Total & 94 & $100,00 \%$ \\
\hline
\end{tabular}

Fonte: Autores. 
Tabela 6 - Manifestações locais e sistêmicas identificadas nos 94 casos de acidentes ofídicos ocorridos na Ilha de Colares - PA, de janeiro de 2007 a dezembro de 2011 notificados ao SINAN

\begin{tabular}{ccc}
\hline Manifestação local & Valor absoluto & Percentual \\
\hline Dor & 87 & $92,55 \%$ \\
Edema & 61 & $64,89 \%$ \\
Equimose & 1 & $1,06 \%$ \\
Necrose & 1 & $1,06 \%$ \\
Outra & 1 & $1,06 \%$ \\
\hline Manifestação sistêmica & Valor absoluto & Percentual \\
\hline Vagais & 3 & $3,19 \%$ \\
Neuroparalíticas & 2 & $2,13 \%$ \\
Hemorrágicas & 2 & $2,13 \%$ \\
Miolíticas & 1 & $1,06 \%$ \\
\hline
\end{tabular}

Fonte: Autores.

Em 83 fichas, informações quanto à evolução do caso das vítimas foram preenchidas, sendo que destas a maioria evoluiu para cura $(82,98 \%$; $\mathrm{n}=78$ ), mas em cinco esse parâmetro foi ignorado, demonstrando certa displicência quanto ao desfecho do acompanhamento clínico dos pacientes. Além disso, outras falhas graves foram identificadas neste estudo, principalmente quanto ao preenchimento de informações importantes, tais como a ocupação da vítima e o tipo de serpente envolvida no acidente.

\section{Discussão}

De acordo com Guimarães (2015) o município de Colares apresenta atividades econômicas centradas principalmente no extrativismo animal, vegetal e agricultura familiar caracterizada especialmente pelos roçados de mandioca (Manihot esculenta) em áreas geralmente preparadas com a queima da vegetação rasteira para posterior plantio das culturas. Estas características devem ser consideradas quando se estuda ofidismo em uma determinada região, pois a aproximação do homem com a natureza e com atividades agrícolas aumenta os riscos de ocorrência de acidentes ofídicos (BOCHNER; STRUCHINER, 2003; LIMA; CAMPOS; RIBEIRO, 2009, LIMA et al., 2009; MORENO et al., 2005; NASCIMENTO, 2000; PINHO; OLIVEIRA; FALEIROS, 2004; SOUZA et al., 2013). Sendo assim, o exercício desta atividade pode ser tomado como fator de risco para a ocorrência de acidentes com serpentes peçonhentas, uma vez que, de acordo com os dados, a maioria dos acidentes ocorreu em indivíduos do sexo masculino e durante a realização do trabalho. Além disso, estas informações reforçam a conotação do acidente ofídico como acidente de trabalho, uma vez que o seu incremento coincide com o deslocamento do trabalhador rural para as atividades do campo (MISE; LIRA-DA-SILVA; CARVALHO, 2007).

Como observado, houve um ligeiro aumento no número de notificações de janeiro de 2007 a dezembro de 2011, fato que pode ser explicado por dois fatores. O primeiro se refere ao desmatamento para o desenvolvimento de áreas de plantio agrícola na região, resultando em alterações ambientais e, consequentemente, em maior mobilidade de serpentes de fragmentos florestais para a área urbana da ilha (IDESP, 2013; PINHO; OLIVEIRA; FALEIROS, 2004). Já o segundo, se refere à melhoria no sistema de notificação e à maior facilidade de acesso dos usuários aos postos de atendimento de saúde, quadro que vem sendo observado em várias regiões do Brasil ao longo dos anos e que pode ter influenciado no aumento das notificações encaminhadas ao Ministério da Saúde, mas não necessariamente no número de casos (FISZON; BOCHNER. 2008). 
De acordo com a Fundação Nacional de Saúde, a distribuição mensal dos acidentes na região Norte não apesenta sazonalidade marcante, ocorrendo casos uniformemente durante todo o ano (ARAÚJO; SANTALUCIA; CABRAL, 2003; BRASIL, 1998). No entanto, alguns estudos vêm revelando clara relação entre o índice pluviométrico mensal da região e os meses com maior frequência de acidentes (LIMA; CAMPOS; RIBEIRO, 2009; MORENO et al., 2005; NASCIMENTO, 2000; WALDEZ; VOGT, 2009). Esta diferença na distribuição mensal dos casos pode ser explicada devido às características biológicas das serpentes, pois, alguns gêneros de ofídios amazônicos, estão mais ativos na estação chuvosa que, para a área estudada, compreende os seis primeiros meses do ano (BERNARDE; ABE, 2006; BERNARDE; ALBUQUERQUE; TURCI, 2012; BRASIL, 2009; SILVA et al., 1999a, 1999b; TURCI et al., 2009), coincidindo com os picos de umidade relativa do ar (SILVA et al., 1999a, 1999b). Com o aumento do índice pluviométrico ocorre transbordamento de corpos d'água (leito de rios, igarapés e açudes), fazendo com que as serpentes procurem por terra firme e, assim, aumentando a possibilidade de encontro com a população (BERNARDE; ABE, 2006; OLIVEIRA; MARTINS, 2001). Além disso, Oliveira e Martins (2001) sugerem, ainda, que os padrões de atividade da população e das serpentes podem estar relacionados com a intensificação dos envenenamentos durante a época de cheia.

Quanto à zona de ocorrência dos acidentes, verificou-se que a maioria ocorreu na zona rural, resultado já esperado devido às características socioeconômicas do município e aos fatores de risco associados ao meio agrícola, tal como encontrado em outros estudos que reforçam a relação entre acidentes ofídicos e campo (LEITE et al., 2013; MISE; LIRA-DA-SILVA; CARVALHO, 2007; MORENO et al., 2005; SOUZA et al., 2013). No entanto, vale ressaltar que apesar da baixa frequência, os casos registrados em áreas urbanas requerem especial atenção, pois podem indicar a necessidade de infraestrutura básica e adequada para manutenção destes animais em sinantropização, como afirmam Lima, Campos e Ribeiro (2009). Tal ressalva reside no fato de que na zona urbana, o aumento na quantidade de resíduos domésticos produzidos e acondicionados de forma precária, principalmente nas áreas onde estão os bolsões de pobreza, atrai pequenos roedores cujas principais predadores são serpentes (LIMA-VERDE, 1994).

Com relação à ocupação das vítimas, mais da metade das fichas não tinham essa informação preenchida, viés que pode prejudicar a interpretação dos dados quantitativa e qualitativamente. Entre as ocupações registradas, os estudantes foram os principais envolvidos nos acidentes ofídicos, tal como observado por Moreno et al. (2005) e Lima, Campos e Ribeiro (2009). Essa grande frequência pode estar relacionada ao ir e vir da escola, uma vez que devido à distância entre a instituição e a moradia e à deficiência de transporte apropriado, os estudantes das zonas rurais enfrentam longas caminhadas, geralmente passando por áreas de risco (MORENO et al., 2005). Além disso, é comum os jovens do meio rural auxiliarem seus pais em atividades agrícolas, fato que pode ter contribuído para o elevado índice dessa categoria nos resultados encontrados.

A faixa etária mais acometida está compatível com os dados da literatura nacional e regional (BERNARDE; GOMES, 2012). A explicação para tais achados reside na relação existente entre o sexo masculino e a idade economicamente ativa (21 - 40 anos de idade), caracterizada geralmente por chefes de família cuja principal fonte de renda advém do trabalho no campo (LIMA; CAMPOS; RIBEIRO, 2009). A zona rural, especialmente as áreas de plantio são os locais de maior ocorrência de envenenamentos por serpentes e, por conta do perfil geral dos trabalhadores rurais, justificam-se os achados do presente trabalho (SARAIVA et al., 2012). 
As crianças estão inseridas no segundo grupo mais acometido ( 0 - 20 anos de idade), provavelmente devido à maior vulnerabilidade dessa faixa etária em virtude da menor capacidade de se defender ou do desconhecimento do perigo representado pelos animais peçonhentos. É importante destacar que esta faixa etária concentra também os indivíduos que estão começando a exercer, de forma precoce, o trabalho agrícola, muito provavelmente com vistas a contribuir para o aumento da renda familiar, como observado por Bochner e Struchiner (2004), o que aumenta os riscos deste grupo (crianças e jovens) de ser vitimado por animais peçonhentos. Finalmente, os indivíduos menos acometidos são os mais velhos (acima de 60 anos), provavelmente devido à baixa atividade de pessoas sexagenárias e, portanto, a menor exposição dos idosos aos fatores de risco, tal como observado por Nascimento (2000).

A maioria dos acidentes foi provocada por serpentes do gênero Bothrops, resultado que está de acordo com o padrão observado em outras regiões do Brasil (BOCHNER; STRUCHINER; 2004; LEITE et al., 2013), inclusive na Amazônia (BERNARDE; ABE, 2006; BERNARDE; GOMES, 2012; TURCI et al., 2009) e, segundo Campbell e Lamar (2004), a espécie Bothrops atrox é a responsável pela maioria deles. Acidente laquético foi o segundo mais frequente corroborando com Nascimento (2000), o qual afirma que, embora serpentes do gênero Lachesis estejam presentes em toda a Amazônia, ocasiona raros acidentes nas áreas em que habitam.

Em relação ao tempo decorrido entre a picada até o atendimento ambulatorial das vítimas, sabe-se que a administração precoce de soro heterólogo, assim como a especificidade, quantidade e via adequada de administração são fatores determinantes na evolução dos casos de envenenamentos (MORENO et al., 2005). Portanto, o tempo tem grande importância para o prognóstico do acidentado, pois quanto menor esse tempo, menores são as chances de ocorrerem complicações como necrose, síndrome compartimental e insuficiência renal (CAIAFFA et al., 1997). Quanto a esse parâmetro, verificou-se que na maioria dos acidentes, o tempo decorrido entre a picada até o atendimento ambulatorial foi inferior a uma hora, fato que sugere um atendimento relativamente rápido.

As falhas quanto à administração de soro antiofídico identificadas no presente estudo, como a sua não administração, o emprego de terapia inespecífica e a sua administração em dose abaixo da recomendada pelo Ministério da Saúde (10 ampolas para acidentes laquéticos) (BRASIL, 2001), por exemplo, já foram relatadas em outras publicações em várias regiões do Brasil (MORENO et al., 2005; SOUZA et al., 2013) e só retratam claramente as fragilidades no sistema de atendimento ao paciente acidentado, prejudicando a qualidade do atendimento ou, no mínimo, caracterizam displicência da equipe de saúde quanto ao preenchimento da ficha.

Em relação à região anatômica acometida durante os acidentes ofídicos, os membros inferiores foram os mais atingidos, sendo o pé e a perna as áreas mais afetadas, resultado que sugere o uso de indumentária inadequada e corrobora com outros estudos (LIMA; CAMPOS; RIBEIRO, 2009; LIMA et al., 2009; MORENO et al., 2005; SARAIVA et al., 2012; SOUZA et al., 2013). Sabe-se que a ausência de utilização de equipamentos de proteção individual, tais como botas de cano longo, perneira, e demais vestimentas tornam os trabalhadores mais susceptíveis aos ataques nessas áreas (membros inferiores) (BOCHNER; STRUCHINER, 2003; LIMA; CAMPOS; RIBEIRO, 2009; LIMA et al., 2009). Além disso, outro fator que deve ser levado em consideração é que as serpentes peçonhentas são répteis terrestres que possuem a habilidade de atacar as presas por meio de botes, o que contribui para a maior ocorrência de ataques aos membros inferiores quando comparados aos membros superiores (D'AGOSTINI; CHAGAS; BELTRAME, 2011). Em todo caso, atenção no campo é a principal medida preventiva, porque uma serpente pode estar em qualquer lugar, desde o chão ou sobre a vegetação, imóvel camuflada ou se deslocando (BERNARDE, 2012). 
Na maioria dos acidentes os sinais clínicos foram alterações locais, caracterizada principalmente por dor e edema, enquanto que as manifestações sistêmicas se restringiram a alterações vagais, neuroparalíticas, hemorrágicas e miolíticas, compatíveis com quadro clínico assinalado na literatura (ALBUQUERQUE; COSTA; CAVALCANTI, 2004; BRASIL, 2001).

A falha quanto ao preenchimento de informações importantes nas fichas de notificação demonstra claramente uma fragilidade na metodologia adotada em esfera municipal e federal, também apresentada em outros estudos (SARAIVA et al., 2012) e, associadaaos constantes casos de subnotificações que seguem na região Norte (ARAÚJO; SANTALUCIA; CABRAL, 2003), denunciam falhas no sistema de notificação e apontam para urgentes medidas para melhorias nos sistemas utilizados atualmente, tanto em relação aos registros, quanto aos procedimentos terapêuticos adotados para que haja melhoria da promoção da saúde da população.

\section{Conclusões}

Este estudo possibilitou identificar que, assim como em outras regiões amazônicas, a população rural do município de Colares também está sujeita a casos de acidentes ofídicos, principalmente em se tratando de trabalhadores rurais ou aquelas pessoas com atividades ligadas ao campo. Além disso, o estudo demonstrou claramente que, em Colares, existem falhas quanto ao preenchimento das fichas de notificação e à terapia instituída nos casos estudados. Portanto, medidas emergenciais devem ser tomadas a fim de melhorar a qualidade do atendimento e das informações, visto que se trata de um problema de saúde pública e de notificação obrigatória. Sendo assim, sugere-se que haja um treinamento da equipe de saúde local, bem como a realização de estudos etnozoológicos associados a estudos epidemiológicos transversais, para que seja feita uma avaliação mais fiel da situação epidemiológica da localidade.

\section{Agradecimentos}

À CAPES pela concessão da bolsa de mestrado e pelo apoio financeiro, por meio do Edital PROCADNF UFRA-UFRPE-UNESP Botucatu. Aos funcionários da Secretaria de Saúde do Município de Colares pela disponibilidade dos dados e de apoio técnico. Aos pesquisadores envolvidos no estudo, em especial à Prof ${ }^{a}$ Dora, minha orientadora.

\section{Referências}

ALBUQUeRQuE, H. N.; COSTA, T. B. G.; CAVALCANTI, M. L. F. Estudo dos acidentes ofídicos provocados por serpentes do gênero Bothrops notificados no estado da Paraíba. Revista de Biologia e Ciências da Terra, Paraíba, v. 5, n. 1, p. 1-8, 2004.

ARAÚJO, F. A. A.; SANTALUCIA, M.; CABRAL, R. F. Epidemiologia dos acidentes por animais peçonhentos. In: CARDOSO, J. L. C.; SIQUEIRA FRANÇA, F. O.; WEN, F. H.; SANT'ANA MALAQUE, C. M.; HADDAD, V. J. (Ed.). Animais peçonhentos no Brasil: biologia, clínica e terapêutica dos acidentes. São Paulo: Sarvier, 2003. p. 6-9.

BERNARDE, P. S. Anfibios e répteis: introdução ao estudo da herpetofauna Brasileira. São Paulo: Anolis Books, 2012.

. Serpentes peçonhentas e acidentes ofidicos no Brasil. São Paulo: Anolis Books, 2014.

BERNARDE, P. S.; ABE, A. S. A snake community at Espigão do Oeste, Rondônia, Southwestern Amazon, Brazil. South American Journal of Herpetology, São Paulo, v. 1, n. 2, p. 102-113, 2006.

BERNARDE, P. S.; ALBUQUERQUE, S.; TURCI, L. C. Serpentes peçonhentas e acidentes ofidicos em Rondônia. São Paulo: Anolis Books, 2012.

BERNARDE, P. S.; GOMES, J. O. Serpentes peçonhentas e ofidismo em Cruzeiro do Sul, Alto Juruá, Estado do Acre, Brasil. Acta Amazônica, Manaus, v. 42, n. 1, p. 65-72, 2012. 
BOCHNER, R.; STRUCHINER, C. J. Aspectos ambientais e sócio-econômicos relacionados à incidência de acidentes ofídicos no Estado do Rio de Janeiro de 1990 a 1996: uma análise exploratória. Cadernos de Saúde Pública, v. 20, n. 4, p. 976-985, 2004.

- Epidemiologia dos acidentes ofídicos nos últimos 100 anos no Brasil: uma revisão. Cadernos de Saúde Pública, Rio de Janeiro, v. 19, n. 1, p. 7-16, jan./fev, 2003.

BRASIL. Ministério da Saúde. Manual de diagnóstico e tratamento de acidentes por animais peçonhentos. Brasília: Fundação Nacional de Saúde, 1998.

- Manual de diagnóstico e tratamento de acidentes por animais peçonhentos. Brasília, 2001.

- Secretaria de Vigilância em Saúde. Zoonoses: situação epidemiológica das zoonoses de interesse à saúde pública. Boletim Epidemiológico Eletrônico, Brasília, ano 9, n. 1, jun. 2009.

CAIAFFA, W. T.;ANTUNES, C.M.F. OLIVEIRA, H. R.; DINIZ, C. R. Epidemiological and clinical aspects of snakebite in Belo Horizonte, Southeast Brazil. Revista do Instituto de Medicina Tropical de São Paulo, v. 39, n. 2, p.113-118, 1997.

CAMPBELL, J. A.; LAMAR, W. W. The venomous reptiles of the western hemisphere. Ithaca: Cornell University Press, 2004.

D'Agostini, F. M.; CHAGAS, F. B.; BELTRAME, V. Epidemiologia dos acidentes por serpentes no município de Concórdia, SC no período de 2007 a 2010. Evidência - Ciência e Biotecnologia, Joaçaba, v. 11, n.1, p. 51-60, 2011.

FISZON, J. T.; BOCHNER, R. Subnotificação de acidentes por animais peçonhentos registrados pelo SINAN no Estado do Rio de Janeiro no período de 2001 a 2005. Revista Brasileira de Epidemiologia, São Paulo, v. 11, n. 1, p. 114-127, 2008.
GUIMARÃES, C. D. O. A herpetofauna de colares: identificação de taxa, etnozoologia e acidentes ofídicos ocorridos em Colares, Pará, Amazônia oriental. 2015. 134 f. Dissertação (Mestrado em Saúde e Produção Animal na Amazônia) Universidade Federal Rural da Amazônia, 2015.

GUTIÉRREZ, J. M.; HIGASHI, H. G.; WEN, F. H.; BURNOUFOUF, T. Strengthening antivenom production in Central and South American public laboratories: report of a workshop. Toxicon, Oxford, v. 1, n. 49, p. 30-35, 2007.

GUTIÉRREZ, J. M.; THEAKSTON, R. D. G.; WARREL, D. A. Confronting the neglected problem of snake bite envenoming: the need for a global partnership. PLOS Medicine, San Francisco, v. 3, n. 6, p.727-731, 2006.

IDESP - INSTITUTO DE DESENVOLVIMENTO ECONÔMICO, SOCIAL E AMBIENTAL DO PARÁ. Estatística municipal: Município de Colares. 2013. Disponível em: <http://www.idesp.pa.gov.br/ paginas/produtos/EstatisticaMunicipal/pdf/Colares. pdf>. Acesso em: 26 out. 2013.

LEITE, R. S.; TARGINO, I. T. G.; LOPES, Y. A. C. F.; BARROS, R. M.; VIEIRA, A. A. Epidemiology of snakebite accidents in the municipalities of the state of Paraiba, Brazil. Ciência \& Saúde Coletiva, Rio de Janeiro, v. 18, n. 5, p. 1463-1471, 2013.

LIMA, A.C. S. F.; CAMPOS, C. E. C.; RIBEIRO, J. R. Perfil epidemiológico de acidentes ofídicos do estado do Amapá. Revista da Sociedade Brasileira de Medicina Tropical, Uberaba, v. 42, n. 3, maio/ jun, p. 329-335, 2009a.

LIMA， J. S.; MARTELLI JÚNIOR, H. M.; MARTELLI, D. R. B.; SILVA, M. S.; CARVALHO, S. F. G.; CANELA, J. R.; BONAN, P. R. F. Perfil dos acidentes ofídicos no norte do Estado de Minas Gerais, Brasil. Revista da Sociedade Brasileira de Medicina Tropical, Uberaba, v. 42, n. 5, p. 561-564, 2009b.

LIMA-VERDE, J. S. Por que não matar as nossas cobras. In: NASCIMENTO, L. B.; BERNARDES, A. T.; COTTA, G. A. (Ed.). Herpetologia no Brasil 1. Belo Horizonte: Pontifica Universidade Católica/ Biodiversitas, 1994. p. 92-101. 
MISE, Y. F.; LIRA-DA-SILVA, R. M.; CARVALHO, F. M. Envenenamento por serpentes do gênero Bothrops no Estado da Bahia: aspectos epidemiológicos e clínicos. Revista da Sociedade Brasileira de Medicina Tropical, Uberaba, v. 40, n. 5, p. 569-573, set./out. 2007.

MORENO, E.; ANDRADE-QUEIROZ, M.; LIRA-DA-SILVA, R.; TAVARES-NETO, J. Características clínico-epidemiológicas dos acidentes ofídicos em Rio Branco, Acre. Revista da Sociedade Brasileira de Medicina Tropical, Uberaba, v. 38, n. 1, p. 15-21, jan./fev. 2005.

NASCIMENTO, S. P. Aspectos epidemiológicos dos acidentes ofídicos ocorridos no estado de Roraima, Brasil, entre 1992 e 1998. Cadernos de Saúde Pública, Rio de Janeiro, v. 16, n. 1, p. 271276, jan./mar. 2000.

OLIVEIRA, M. E.; MARTINS, M. When and where to find a pitviper: activity patterns and habitat use of the lancehead, Bothrops atrox, in central Amazonia, Brazil. Herpetological Natural History, Stanford, v. 8, n. 2, p. 101-110, 2001.

PINHO, F. M. O.; OLIVEIRA, E. S.; FALEIROS, F. Acidente ofídico no estado de Goiás. Revista da Associação Médica Brasileira, São Paulo, v. 50, n. 1, p. 93-96, 2004.

SARAIVA, M. G.; OLIVEIRA, D. S.; FERNANDES FILHO, G. M. C.; COUTINHO, L. A. S. A.; GUERREIRO, J. V. Perfil epidemiológico dos acidentes ofídicos no estado da Paraíba, Brasil, 2005 a 2010. Epidemiologia e Serviço de Saúde, Brasília, v. 21, n. 2, p. 449-456, jul./set. 2012.

SILVA, J. M. L.; GAMA, J. R. N. F.; VALENTE, M. A.; RÊGO, R. A.; RODRIGUES, T. E.; SANTOS, P. L.; CARDOSO JUNIOR, E. Q.; SILVA, P. R. O. Levantamento de solos de alta intensidade e mapeamento da cobertura vegetal e uso da terra do município de Colares- Estado do Pará. Belém: Embrapa Amazônia Oriental, 1999a.

- Avaliação da aptidão agrícola das terras do município de Colares - Estado do Pará. Belém: Embrapa Amazônia Oriental, 1999b.
SOUZA, S. S.; COSTA, K. M. F. M.; CÂMARA, I. M. B.; MOURA, E. S. R.; FONSECA, Z. A. A. S.; MOREIRA, J. O.; LEITE, A. I. Aspectos epidemiológicos dos acidentes ofídicos no município de Mossoró, Rio Grande do Norte, no período de 2004 a 2010. Revista de Patologia Tropical, Goiânia, v. 42, n. 1, p. 105-113, jan./mar. 2013.

TURCI, L.C. B.; ALBUQUERQUE, S.; BERNARDE, P. S.; MIRANDA, D. B. Activity, habitat use, and behavior of the Bothriopsis bilineatus and of the Bothrops atrox (Serpentes: Viperidae) in Moa river forest, Acre - Brazil. Biota Neotropica, Campinas, v. 9, n. 3, p. 197-206, 2009.

WALDEZ, F.; VOGT, R. C. Aspectos ecológicos e epidemiológicos de acidentes ofídicos em comunidades ribeirinhas do baixo rio Purus, Amazonas, Brasil. Acta Amazonica, Manaus, v. 39, n. 3, p. 681-696, 2009.
Recebido em: 16 fev. 2015. Aceito em: 11 ago.2015. 
